\title{
Revisión de casos internacionales del desempeño de los mercados del agua como punto de referencia a su posible implementación en Colombia
}

Fecha de recepción: 14 de febrero de 2011
Fecha de aprobación: 9 de junio de 2011

\author{
Andrés Chavarro Velandia \\ achavarr@poli.edu.co \\ Politécnico Grancolombiano
}

Economista de la Universidad Nacional de Colombia, magíster (C) en Economía de la Universidad Externado de Colombia. Es profesor investigador del Politécnico Grancolombiano y miembro del Grupo de investigación Empresa, Economía y Globalización del Politécnico Grancolombiano.

\section{Resumen}

Desde hace unos diez años, en varios países de América Latina, se ha venido presentando la tendencia a establecer leyes sobre el recurso hídrico. Éste ha sido el caso reciente de Perú (2009), Ecuador (2010), Bolivia (2010) y Colombia (2005 y 2008), entre otros países. En algunos de estos casos se abre la posibilidad de establecer los mercados de agua como una de las herramientas para gestionar el recurso, y en otros casos, como el colombiano, si bien no se establecen taxativamente, tampoco niega la posibilidad de que se puedan desarrollar.

El presente artículo compila y estudia algunas de las experiencias más sobresalientes en la implementación de mercados de agua a nivel internacional. A partir de clasificar los casos estudiados en dos grupos (mercados formales e informales), se establecieron sus principales características al identificar cuáles son sus principales fortalezas, debilidades y condiciones mínimas para su desempeño adecuado. Se encontró que los mercados de agua son instrumentos para gestionar el recurso en entornos de escasez relativa, dada la creciente demanda del sector urbano y el deterioro de las fuentes. Su buen desempeño depende significati-

\begin{abstract}
Karla Triana
kntriana@gmail.com

Politécnico Grancolombiano

Profesional de Negocios Internacionales del Politécnico Grancolombiano. Hace parte del semillero de investigación del proyecto Evaluación de la conveniencia de la implementación de los mercados del agua en Colombia como herramienta de gestión del recurso hídrico.
\end{abstract}

\footnotetext{
Abstract

For about ten years, there has been the tendency to establish laws about water resource in several countries of Latin America. This has been the recent case of Peru (2009), Ecuador (2010), Bolivia (2010), and Colombia (2005 and 2008), among other countries. In some of these cases there is the possibility to establish water markets as a tool to manage the resource. In other cases, such as in Colombia, it is not established specifically, but it does not deny the possibility to develop it. This article compiles and studies some of the most outstanding experiences within the implementation of water markets at international level. From classifying the two studied cases in two groups (formal and informal markets) it was possible to establish their main characteristics, strengths, weaknesses, and minimum conditions for their correct performance. We found that water markets are instruments used to manage the resource where there are water shortages due to the increasing demand of the urban sector and the damage of its sources. The performance of these markets depends significantly on the presence of institutions (game rules) that limit the appearance of externalities
} 
vamente de la presencia de instituciones (reglas de juego) que limiten la aparición de externalidades y disminuyan la posibilidad de posibles conflictos distributivos.

\section{Palabras clave}

Mercados de agua, instituciones formales e informales, gestión del recurso hídrico, externalidades. and diminish the possibility of distributive conflicts.

\section{Keywords}

Water Markets, Formal and Informal Institutions, Water Resource Management, Externalities. 


\section{Introducción}

En varios países de América Latina, desde hace unos diez años se ha venido presentando la tendencia a establecer leyes sobre el recurso hídrico. Éste ha sido el caso reciente de Perú (2009), Ecuador (2010), Bolivia (2010) y Colombia (2005 y 2008), entre otros países. En algunos de estos casos se abre la posibilidad de establecer los mercados de agua como una de las herramientas para gestionar el recurso, y en otros casos, como el colombiano, si bien no se establecen taxativamente, tampoco niega la posibilidad de que se puedan desarrollar.

Este interés se ha presentado en tanto la economía de los recursos hídricos en varios países de América Latina ha llegado a una fase madura de oferta inelástica de nuevos recursos e interdependencias crecientes entre los usos y los usuarios del agua. También ocurre que los diseñadores de la política se concentran principalmente en expandir y subsidiar el desarrollo del sector en la generación de ingresos, la gestión de la demanda y la reasignación (Lee \& Juravlev, 1998). Finalmente, los precios como instrumentos de asignación se presentan como atractivos, pues se cree que permitir el comercio de agua genera beneficios que cubren el costo de oportunidad de mantener los niveles de eficiencia dados por las instituciones reguladoras originales (Lee \& Juravlev, 1998).

Más allá de nuestro subcontinente, los mercados de agua han servido generalmente para reasignar excesos de agua usada en el sector agrícola hacia el sector urbano (residencial e industrial) o hacia otros sectores agrícolas que revelan que lo necesitan más. Los resultados de su implementación en tal contexto son en la mayoría de los casos positivos. Sin embargo, no se observa que se apliquen a problemas de asignación distintos. En todo caso, eso sí, han estado acompañados de instituciones formales o informales que se crearon para mitigar los problemas de externalidades que se generan o posibles conflictos distributivos.

El propósito del presente artículo es identificar las experiencias más relevantes en cuanto a la aplicación de los mercados de agua a nivel internacional, de manera que sirva como espejo para el caso de nuestro país, en el que, si bien aún no existe lo que se conoce mundialmente como la ley de aguas (que normativiza alrededor del mercado de agua), es posible que en un momento dado se pueda presentar, por lo que conocer de antemano sus virtudes, flaquezas y condiciones de implementación se hace necesario.

Este artículo es parte de los resultados del proyecto de investigación denominado "Oportunidades para Colombia del uso de mercados de agua", cuyo objetivo es establecer la conveniencia del uso de este instrumento de gestión del recurso en nuestro país a la luz de varios criterios, entre los que se encuentra la dimensión legal, física (disponibilidad), social (usos múltiples del agua), el criterio de expertos y, por supuesto, la experiencia internacional. El proyecto se inició en el año 2010 por el grupo de investigación Empresa, Economía y Globalización del Politécnico Grancolombiano.

El artículo está organizado de la siguiente manera. En la sección 2, en términos generales, se revisa qué son y cómo funcionan los mercados de agua, para definir un conjunto de términos que serán usados a lo largo del texto. En las secciones 3 y 4 se abordan los casos 
encontrados por grupos; primero (sección 3), los mercados llamados informales, en tanto surgen espontáneamente con muy poca o ninguna intervención del gobierno; luego (sección 4), se examinan los casos de mercados formales; por último y a manera de conclusión, en la sección 5 , se consolidan los principales hallazgos de la revisión realizada, considerándolos desde la perspectiva como enseñanza.

\section{2. ¿Qué son y cómo funcionan los mercados del agua?}

Uno de los primeros elementos que legitiman hablar de mercados de agua es que de acuerdo a los principios de $\mathrm{Du}$ blin (1992) en el marco de la conferencia internacional sobre el agua y el medio ambiente, esta se considera como un bien económico: el agua tiene un valor económico en todos sus diversos usos en competencia a los que se destina y debería reconocérsele como un bien económico. De hecho, se ha discutido esa posibilidad desde los años sesenta, no obstante son otros los caminos que han recorrido las administraciones en la gestión de los recursos hídricos (Lee \& Juravlev, 1998).

Los mercados de agua como herramienta de gestión del recurso significa el intercambio de derechos de agua natural voluntaria entre compradores y vendedores. Los mercados de agua sirven para adjudicar total o parcialmente del recurso agua entre agentes bajo el principio de la transferibilidad. En tanto los individuos poseen la propiedad privada sobre el agua, están en disposición de cambia agua por dinero $u$ otra propiedad. Teóricamente estos derechos son independientes de la tierra sobre la cual ellos se usan significando que el agua puede ser intercambiada separadamente de la tierra (Griffin, 2006, p. 204).

Hay un grupo de por lo menos seis mecanismos de transferencia de agua (Hadjigeorgalis, 2009). El primero es el banco de agua, el cual es una institución central que enlaza a usuarios interesados en comprar o vender agua. El agua es vendida a un costo que incluye un margen adicional y que cubre los costos de operación del banco, los cuales son pagados usualmente por el comprador. Los bancos de agua pueden ser instituciones temporales o permanentes. Otra opción, es el Bulletin Board Markets que a menudo se conoce también como bancos de agua; en este caso el precio no lo da una institución central. En cambio, los compradores y vendedores intercambian ofertas y requerimientos de agua o derechos de agua en una especie de página de clasificados ubicados usualmente en las oficinas centrales del distrito de riego o a través de plataformas electrónicas. También pueden encontrarse de doble subasta, en los cuales compradores y vendedores se someten a propuestas de sobre sellado para volúmenes de agua. Hay un operador de intercambio que agrega todas las propuestas y los precios de venta para determinar un precio conjunto de mercado que asigne la mayor parte de agua.

En cuanto a las operaciones que involucren suministro futuro, están los mercados de derivados en los que se hacen contratos de opciones o futuros. Los contratos futuros hacen que el comprador pague por el agua sobre la fecha del contrato definida previamente junto con el precio. Por último, se encuentran las figuras de leasing ambiental o programas de gasto que permiten el acceso al agua 
de riego que incrementa el flujo de corriente para la protección de peces o de vida salvaje. Aquí, hacen parte estrategias como la de leasing público y la compra de derechos de riego y programas de recompra.

¿Cómo funciona en términos esenciales el comercio de agua? Un esquema sencillo fue desarrollado por Griffin (2006). En principio hay que suponer que los derechos de agua son propiedad privada $y$ hay por dos agentes que tienen un valor marginal del agua diferente lo que los hace intercambiar entre ellos. Cada agente posee una cantidad $\mathrm{w}_{1} \mathrm{y} \mathrm{w}_{2}>0$ de derechos transferibles de agua. Además los agentes tienen funciones lineales de beneficios marginales netos de la forma:

$\mathrm{MNB}_{1}=\mathrm{b}_{1}-\mathrm{m}_{1} \mathrm{w}_{1}$
$\mathrm{MNB}_{2}=\mathrm{b}_{2}-\mathrm{m}_{2} \mathrm{w}_{2}$

Digamos que w1 y w2 toman valores particulares w1'y w2' y reemplacémoslas en (1)

Si $\mathrm{MNB}_{1}{ }^{1} \mathrm{MNB}_{2}$ hay ganancias para ambos de un posible comercio siempre y cuando los costos de transacción no sean prohibitivos. Supóngase que $\mathrm{MNB}_{1}$ $>\mathrm{MNB}_{2}$. En este caso, el agente 2 debe ceder algo de agua al agente 1 no porque sea económicamente eficiente, sino porque es ventajoso para él. ¿Cuánta agua deben comerciar los agentes y a qué precio? En ausencia de costos de transacción los agentes pueden maximizar sus beneficios individuales en el punto en el que

$$
\mathrm{MNB}_{1}=\mathrm{MNB}_{2}
$$

Pero además se debe garantizar que la cantidad de agua a asignar no sea ni inferior ni superior a la cantidad de agua disponible. Así la restricción queda: $\mathrm{w} 1^{\prime}+\mathrm{w} 2^{\prime}=\mathrm{w} 1^{*}+\mathrm{w} 2^{*}$

donde $\mathrm{w}^{*}$ y w2* son los valores óptimos de derechos de agua que deben tener cada agente.

Combinando (2) y (3) la cantidad de agua transferida será w2' - w2*. En el margen, cada unidad de agua será valorada de la misma manera por los agentes después de la transferencia.

Gráficamente este modelo se puede ver de la siguiente manera:

Figura 1. Comercio de agua entre dos agentes en ausencia de costos de transacción.

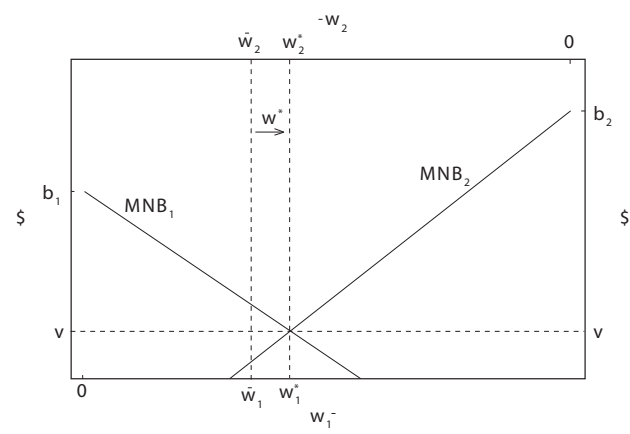

Fuente: tomado de Griffin 2006, p. 209.

Ahora bien, hay por lo menos tres grandes formas en que los mercados de agua se pueden configurar: la aproximación de precio, los mercados de agua informales y los mercados de agua formales (Thobani, 1998).

La primera, consiste en que la autoridad pública procure que el precio que el usuario del agua paga refleje la verdadera escasez o costo de oportunidad. Por ejemplo si el sector agrícola experimenta en excedente de agua de riego que una compañía de la ciudad pudiera tener para su funcionamiento, ésta se encontraría dispuesta a pagar por aquella 
agua. Ello incentivaría a los agricultores a hacer más eficientes sus consumos de agua (o inclusive a cambiar de negocio) para poder aprovechar la posible venta de agua. Si bien el sistema parece razonable no está exento de problemas. Uno de ellos es que puede tener costos de transacción elevados debido a que la autoridad que fija el precio debe tener un sistema para medir y monitorear el flujo de agua, además de que el costo de oportunidad varía de acuerdo a la ubicación, calidad del agua, período del año, disponibilidad y uso.

El otro gran problema de este sistema de precios es el de orden político. En particular, el asunto es que no todos los agricultores estarán convencidos de, por ejemplo, cambiar de fuente de abastecimiento del recurso, porque de un momento a otro le comunicaron que debe usar agua de una fuente más cara y distante, siendo que él informalmente podría servirse de una fuente de un río cercano. En consecuencia, moverán su aparato de cabildeo para evitar cambio institucional (Thobani, 1998).

La segunda forma hace referencia a los mercados informales. Ellos surgen espontáneamente en ausencia de una reacción rápida del Gobierno ante cambios en la demanda. Son técnicamente ilegales pero el Gobierno no los persigue rigurosamente puesto que son de aceptación popular y relocalizan el agua rápida y voluntariamente. Un ejemplo de estos mercados es la situación en la que los agricultores venden un volumen específico de su excedente para un período de tiempo particular a algún vecino, quien a su vez puede vender una parte a algún otro vecino de la localidad. Las principales críticas a este sistema informal son las siguientes: primero, puede haber abusos de posición dominante por ejemplo en el caso en el que un agricultor con suficiente dinero puede generar una red de tuberías tal que le cobre precios de monopolio a pequeños agricultores; segundo y la oportunidad de vender hace que se incremente la explotación de aguas subterráneas deteriorando acuíferos.

Por último, se encuentran los mercados formales. Ellos surgen a través de disposiciones legales que establecen derechos de propiedad transferibles. Su objetivo es reflejar el costo de oportunidad del agua. Su ventaja con respecto a los mercados informales es que son mejor monitoreados y es más fácil hacer cumplir los contratos establecidos. Sin embargo, para que los mercados formales funcionen, se requiere que los usuarios tengan algún tipo de derecho que comprar o vender. Una dificultad inherente es establecer cuántos y a quiénes se les dan derechos inicialmente $y$, todavía peor, esta sesión de derechos a los usuarios hace que las organizaciones públicas asignadoras sientan que pierden poder cada vez que asignan derechos puesto que ya no tienen la discrecionalidad para el suministro bajo sus condiciones, sino que deben hacerlo en los términos de la demanda de los usuarios. Adicionalmente, hay que tener en cuenta que con los mercados formales surgen los costos de transacción en tanto que hay que proteger del daño a terceras partes, generar la suficiente infraestructura para transferir el agua a los compradores e implementar un sistema para hacer cumplir los derechos adquiridos en los contratos (Easter, Dinar, \& Rosengrant, 1998). Los mercados de agua son sólo una de las alternativas para afrontar la creciente escasez del recurso. Ésta se origina por una presión de demanda motivada por el crecimiento 
poblacional localizado en las zonas urbanas o la mayor actividad industrial. De otro lado, la escasez puede deberse a restricciones de la oferta como el agotamiento o la contaminación de las fuentes. Una opción para mitigar la escasez es el uso de otras fuentes como las aguas subterráneas, pero ésta es una alternativa limitada por la contaminación que están experimentando. Si esta opción no es viable puede traerse agua de otros lugares, así sea en contextos geopolíticos conflictivos (Wachtel, 2004) (como en el caso de Israel que trae agua de Turquía), pero hay que considerar que es un esquema costoso y que implica otro sistema de distribución y de asignación. Si este impedimento es prohibitivo se pueden explorar fuentes no tradicionales como la desalinización de aguas marinas que por ahora es muy costosa, o el reuso, que es funcional en la medida en que se aplique en zonas áridas donde encontrar nuevas fuentes sea muy costoso. Por último, existe la posibilidad de usar lo que se conoce como agua de cosecha (water harvesting) que "es la captura y distribución de agua lluvia o producto de inundaciones a campos con el fin de irrigar cultivos" (Easter et al., 1998). Sin embargo, a la luz del análisis económico básico, el problema general de estas alternativas es que no reflejan el real valor de escasez del agua para sus usuarios en la medida en que no se busca ahorro de agua, no se mejora la eficiencia de uso y se mantienen amplios subsidios tanto para el consumo del sector agrícola como para el del urbano.

Si bien es una herramienta útil para revelar el verdadero valor de escasez del agua, los mercados tienen en principio dos dificultades. La primera es que como se basan en un sistema de precios para reflejar la escasez, éste tiende a subir, lo que genera enseguida rechazo por los usuarios tradicionales que usarán presión política para debilitar el sistema. Ahora bien, esta amenaza se puede corregir si se genera un sistema adecuado de incentivos que muestre que se obtendrían ganancias de hacer transferencias entre usuarios. Dicho sistema trae consigo el segundo problema: los costos de transacción propios de su funcionamiento. Estos se generan principalmente en mercados formales, es decir, en aquellos que requieren de un sistema legal que sea garante de su correcto desempeńo. En tal contexto los costos de transacción se agrupan en dos: los inducidos por cuestiones administrativas (AIC en inglés) y los inducidos por la política pública (PIC en inglés). Easter et al., (1998) muestran en un sencillo esquema que es posible que aún después de incluir ambos tipos de costos de transacción se presenten ganancias de realizar intercambios de mercado. En condiciones sin intercambio sólo se asigna $Q_{1}$ (ver figura 1) para el sector urbano y el resto va para la agricultura. La oferta $\mathrm{O}_{1}$ mide los beneficios de los agricultores cuando deciden vender agua. La demanda $\mathrm{D}_{1}$ es la demanda urbana de agua sin costos de transacción. En ausencia de costos de transacción la cantidad eficiente de agua es $\mathrm{Q}_{2}$. Ahora bien, si se dan costos AIC estos son asumidos por la demanda, de modo que ésta se contrae. En tales circunstancias la cantidad asignada es $\mathrm{Q}_{3}$. Pero si, finalmente, también se incluyen los costos PIC, ellos son asumidos por la oferta, por lo que ésta se contrae hasta $\mathrm{S}_{2}$. Es así como en presencia de los dos grupos de costos de transacción, la cantidad asignada finalmente es $\mathrm{Q}_{4}$ que de todas formas es mayor que la cantidad inicial $\mathrm{Q}_{1}$ a un precio menor (para la 
demanda) y con beneficios más grandes para la oferta (los agricultores). En cualquier caso, entonces, el intercambio aumenta el beneficio para todos.

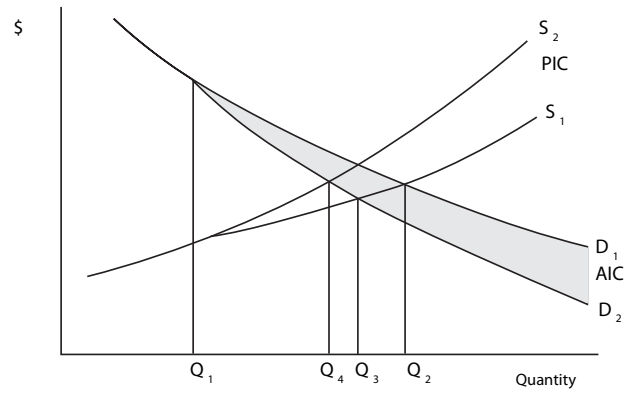

Fuente: tomado de Easter et al., 1998, p. 5.

Otra complicación que tienen los mercados de agua es que no están exentos de fallar en la asignación. Hay tres condiciones necesarias para evitar dichas fallas en la localización eficiente del recurso (Livingston, 1998):

1) Los usuarios del recurso tienen que estar ciertos sobre la cantidad, calidad, ubicación y disponibilidad temporal del recurso.

2) El uso del recurso no debe afectar o ser afectado por la utilización del recurso por otro agente (no presencia de externalidades).

3) Debe ser posible relocalizar el recurso en el tiempo en respuesta a cambios en la condiciones.

El problema consiste en que la naturaleza física del agua hace que el recurso sea propenso a fallas de mercado y a uso ineficiente (Livingston, 1998) debido entre otras razones a que la oferta de agua es incierta. Esto sucede por dos razones: en primer lugar, porque hay una alta variabilidad en el régimen de precipitaciones principalmente en regiones áridas. En segundo lugar, porque el uso de agua es abundante en externalidades (principalmente porque la oferta de agua está concentrada y retirada y es consumida por individuos) y esto con toda seguridad afecta la cantidad, calidad y tiempo de oferta para otros usuarios de agua a lo largo de la corriente o dentro del mismo acuífero.

\section{Experiencias de mercados informales}

Usualmente los mercados informales se ubican en países en desarrollo donde las relaciones de intercambio están menos normadas legalmente. Allí, la forma de hacer cumplir los contratos no es por un sistema legal sino por la reputación, la moralidad general y la confianza personal dentro de las redes sociales.

En una de las naciones más pobladas, la India, caracterizada por la escasez del recurso hídrico, se ha generado una economía que depende en algún grado de la disponibilidad del agua. Dichas condiciones han forjado la creación de pequeños mercados informales de este recurso, que presentan entre dos y ochenta compradores por cada vendedor. Esto da a los segundos, en algunos casos, el control del funcionamiento del mismo tal como sucede en Gujarat y Tamil Nado, en donde se evidencian tendencias monopolísticas y oligopólicas. En ciudades como Andhra Pradesh, Uttar Pradesh, Orissa y West Bengal, se puede decir que hay más condiciones para la competencia (Saleth, 1998).

Dichos instrumentos nacieron cuando cada granjero inició la construcción de su propia infraestructura para abastecer 
sus necesidades, principalmente de riego, con la libertad de hacer uso de más agua de la que necesitaba. Así, se desencadenaron una serie de iniciativas para abastecer con el excedente a las granjas que no contaban con el recurso hídrico necesario ni con los fondos suficientes para extraer éste del subsuelo. Por lo anterior, los mercados fueron desarrollados por el sector privado, quien los administra y controla sin ningún tipo de institución o intervención. En consecuencia, los derechos al agua no son claros y se evidencia que las personas más influyentes tienen prelación (Saleth, 1998).

A lo largo de los años, el Estado ha generado varias políticas afines, tales como la Política Nacional de Aguas en 1987 y la Autoridad Central de Aguas Subterráneas en 1997. Sin embargo, su implementación ha sido nula a excepción de Gujarat y Maharashtra, en donde se han introducido algunas condiciones mínimas. Tal regulación ha sido difícil de construir debido a lo diferentes que son las regiones del país: según el Gobierno de India, se recomienda un mínimo de agua per cápita por día de 70 a 100 litros $y$, sin embargo, hay lugares como Ludhiana, donde cuentan con 205 lpcd. De igual forma, otro aspecto a tener en cuenta según Bathla, es la estrecha relación que existe entre la tasa de crecimiento de la población y la aparición de conflictos por el agua, argumentando lo mencionado anteriormente (Bathla, 2000).

Estos mercados de agua tienen como principal destino la agricultura (riego), sin embargo, también abastecen otro tipo de usos como la construcción en Uttar Pradesh y el uso doméstico urbano en Tamil Nadu. De igual forma, según la región, existe o no discriminación de precios: en
Gujarat la tarifa es la misma para todos los compradores, mientras que en Vaigai Basin y Tamil Nadu, éstas son variables según las necesidades del vendedor. Otro factor que afecta el costo de las transacciones está relacionado con otros recursos que son vitales para aquellas economías como el combustible, la electricidad y el arroz entre otros. De esta manera, si dichos productos se encarecen, de igual forma lo hace el agua (Saleth, 1998).

Para entender un poco más cómo funcionan los mercados del agua y cómo se afectan los costos de las transacciones, es importante conocer cómo se lleva a cabo la contratación. Ejemplo de ello son Panahar y Muidara, donde, como en otros lugares de India, se desarrollan estos mercados a través de dos formas: la primera consiste en la tarifa por tiempo, en la que el usuario paga por cada minuto durante el que el tubo permanece abierto abasteciendo el recurso; y la segunda, relacionada con la superficie cultivada, en la cual se paga una tarifa fija sin importar la cantidad de agua que se necesite. Al utilizar este modelo, en algunos casos, los agricultores son abastecidos con menos de lo que necesitan para producir (Rawal, 2002).

Autores como Saleth (1998), mencionan que estos mercados informales del agua han generado una ganancia importante en materia de eficiencia y productividad en el manejo de este recurso, que se ha visto reflejada en las comunidades participantes a través de empleo y mayores ingresos, incluso para quienes no son terratenientes. Sin embargo, aclara que tales resultados se podrían mejorar formalizando los mercados a través de la creación de instituciones que establezcan reglas claras y equitativas para su funcionamiento. En contraste, otros como Rawal (2002) plantean que se deben analizar otras formas 
de administración del agua que no tengan un interés meramente económico.

De una forma muy similar funcionan los mercados de agua en Pakistán. Este país, uno de los más áridos del mundo, cuenta según un informe del Banco Mundial con las siguientes características: intensa degradación de los ecosistemas, sobreexplotación del agua subterránea y deterioro de su calidad, cambio climático, mínima investigación en tecnologías para el mejor aprovechamiento del recurso hídrico, implementación de sistemas que no son financieramente viables, infraestructura precaria, mínima gobernanza y baja confianza en las instituciones e ineficiente uso del agua en la producción agrícola (Briscoe \& Qamar, 2006).

Teniendo en cuenta las condiciones anteriores, los mercados de agua que han tenido lugar en este país se han caracterizado por su informalidad. Estos nacieron debido al manejo que en un principio dio el Estado al recurso cuando estableció precios muy bajos que fomentaron la creación de una demanda mayor a la oferta. Estos se caracterizaban además, por la adjudicación del agua a través de un sistema rotativo de acuerdo con el tamańo de los terrenos que poseían los agricultores. Sin embargo, había quienes se quejaban de que la entrega del recurso no era muy confiable y, en algunos casos, el ciclo no llegaba al final. Debido a los vicios mencionados anteriormente, el Estado cedió la administración del agua subterránea a empresas privadas; esto dio lugar a que los grandes terratenientes invirtieran el capital necesario para llevar a cabo las obras de infraestructura que se requerían y obtuvieran un poder proporcional a su inversión, de la mano de una mayor ventaja para el aprovechamiento del recurso (Meinzen-Dick, 1998).
Otros casos de mercados informales son los de Suráfrica y China. En el caso de Suráfrica, a partir de la Ley Nacional de Agua de 1998 se reconoce el interés público por la mejor gestión del recurso, que se venía haciendo con escaso desarrollo institucional. Esto se veía reflejado en una my baja claridad legal y administrativa del funcionamiento del mercado, insuficiente información para la resolución de conflictos y desconocimiento de registro o titulación de derechos (Grafton, Landry, Libecap, McGlennon, \& O’Brien, 2010). En términos de eficiencia económica, también se observa la presencia de mercados informales pues no hay adecuada información sobre el tamaño del mercado, las ganancias que deja éste y la proporción promedio de uso. Sin embargo, sobresale en este caso que hay estabilidad en la formación de los precios y una relativa calidad del título adquirido y de la información de la formación del precio de mercado (Grafton et al., 2010).

China por su parte, presenta una situación crítica de escasez de agua en el norte del país, en zonas como la cuenca del río Yangtze y los ríos Huang, Huai y Hai. Tal situación ha generado limitaciones para el desarrollo económico de la región, lo que ha dado lugar al desarrollo de mercados que funcionan de forma muy similar a los demás países asiáticos (WorldBank, 2002). En cuanto a su situación de mercados informales se tiene según Grafton et al. (2010) el siguiente panorama: hay un escaso reconocimiento público de interés por promover una mejor gestión del recurso, lo cual se refleja en que no cumple con los mínimos de capacidad administrativa, claridad legal y capacidad de resolución de conflictos y no posee información en cuanto a registro y 
titulación de derechos. A pesar de lo anterior, en términos de eficiencia económica tiene una relativa estabilidad en la formación de precio. Pero, por lo demás, los mercados de agua en China están prácticamente ocultos pues no hay información en cuanto al tamaño del mercado, las ganancias del comercio ni la tasa de uso promedio (Grafton et al., 2010).

\section{Experiencias de mercados formales}

En el occidente de Estados Unidos, debido al crecimiento en la demanda de agua por parte de los centros urbanos, se ha promovido el desarrollo de un gran número de mercados de agua, cada uno con características y legislaciones particulares. Según Brewer, Glennon, Ker y Libecap (2007), los precios en transferencias de agua son mayores cuando el intercambio es de usos agrícolas a usos urbanos que cuando se llevan a cabo entre granjas, lo que ha generado una tendencia a la generación de mercados direccionados hacia los centros urbanos. En cuanto a la regulación, generalmente el Estado interviene en las transacciones para evitar afectar a terceros (Brewer, Glennon, Ker, \& Libecap, 2007).

En Texas, estos mecanismos funcionan por el sistema de derechos de propiedad, con una marcada diferencia entre el agua superficial y el agua subterránea. En cuanto a la primera, los derechos se han cuantificado y dividido uniformemente a diferencia del Valle del Río Grande, pues en general la escasez no es tan significativa, ya que cuentan con un volumen importante de agua subterránea y la legislación cede la autoridad en la administración del recurso hídrico a los diferentes distritos, lo que ha generado que los que son más influyentes manipulen el mercado. Sin embargo, cuentan con una institución encargada del control de las transacciones: la Comisión para la Conservación de los Recursos Naturales, quien aprueba cada transferencia y está en la obligación de llevar a cabo la publicación de la misma para que aquellos que consideren afectados sus intereses interpongan recursos. En términos generales, se caracteriza por el movimiento del agua de los sectores agrícolas al área urbana (Zegarra, 2004).

En cuanto al agua subterránea, se reglamenta bajo el principio de "absolute ownership", mediante el cual el dueño de la tierra también posee los derechos del agua siempre y cuando la use adecuadamente. En general existen sólo dos formas de mercados relacionadas a este tipo de recurso: la primera corresponde a la venta de la tierra, mediante la cual se venden los derechos al agua; y la segunda, consiste en la extracción y el transporte hacia los vecinos que no poseen el recurso necesario para abastecer sus necesidades. Sin embargo, este último no se ha desarrollado en su totalidad debido a que los terratenientes no se asocian para hacer un uso eficiente de la infraestructura. Otra característica es la intensa intervención estatal en el mercado, lo que se ha visto reflejado en un proceso lento para la maduración de éste, debido a una serie de reglamentaciones que no son realmente útiles (Zegarra, 2004).

En relación al comercio, el mercado y las normas que rigen la gestión del agua en Australia, ésta se caracteriza por factores y costos de producción establecidos según la división política del país y el funcionamiento de la economía en cada estación. De allí que en la parte 
baja de la región Murray, se hable de dos tipos de mercados de agua: uno destinado principalmente al riego de cultivos que puede considerarse permanente y otro denominado temporal, según el tiempo sea de lluvia o de sequía. Se cree que el éxito de este último, está vinculado con la estrecha relación que se presenta entre el precio y la estación climática (Brennan, 2006).

Australia se ha preocupado por estructurar una legislación sobre la extracción, el abastecimiento y el manejo que se le debe dar al mercado del agua para prevenir la escasez, sin efectuar una sobreexplotación del recurso. Todo lo anterior, mediante la denominada "Iniciativa Nacional del Agua" donde todas las áreas interesadas se ven involucradas, con el fin de lograr un desarrollo sostenible en este aspecto y constituir un mercado que promueva la solución a diferentes problemas que se pueden generar en materia de agendas políticas, en situaciones de vulnerabilidad frente a un cambio de gobierno o frente a problemáticas mundiales como el cambio climático (Kare Hussey, 2006).

Chile, país reconocido como el líder a nivel mundial en la implementación de mercados de agua desde la concepción del Código de Aguas de 1981 (regulación que le apuntaba completamente al libre mercado), mediante el cual se le dio a éste recurso un significado netamente mercantil, generó un espacio para los mercados de dos maneras: en la asignación original de los derechos del agua, a través de mecanismos de remate y en la reasignación de las aguas entre particulares.

González (2008), quien realizó un trabajo comparativo entre España, Chile y México en cuanto a las características de mercados de agua formales, estructura la configuración de los mercados de agua usando diez criterios: asignación inicial de derechos transferibles, duración de los derechos, formas de transferencia, sistema de asignación de agua en períodos de escasez, tratamiento de la información de mercado, tratamiento de las externalidades, consideración de los costos de transporte, instrumentos de resolución de imperfecciones de mercado, mecanismos para la resolución de conflictos y restricciones al mercado.

Con base en esa clasificación, plantea que en el caso chileno la asignación inicial de derechos se hace tanto con derechos adquiridos como con subastas, la duración de los derechos puede ser permanente o temporal, el agua se transfiere por compra o arrendamiento la asignación en períodos de escasez es por norma prioritaria y norma proporcional. La información de mercado tiene tres procesos conocidos como: homogenización, posibilidad de intermediación privada y utilización de agencias de transacción. En cuanto a las externalidades en el caso de efectos sobre el propio caudal, se utiliza una política pública reguladora y no se manejan los efectos sobre la zona de origen. La financiación de los costos de transporte es tanto pública como privada, para imperfecciones de mercado se utiliza sanción por no uso y los conflictos se resuelven con tribunales de justicia. No hay restricciones al mercado.

Según Humberto Peña (2004), el instrumento utilizado en el primero fue ineficiente, ya que se favorecieron procesos de restricción de la libre competencia, en cuanto a los segundos, no hubo lugar a la declaración de áreas de racionalización. Sin embargo, ha sido evidente la eficiencia del mercado para la reasignación del 
uso del agua a usos que presentan un mayor grado de beneficio social y económico. De igual manera, refiriéndose al Código de Aguas, manifiesta que existen algunas falencias como no determinar plazos para hacer efectiva la inscripción de los derechos del agua y no establecer como obligación de los terratenientes el hecho de informar si se llevan a cabo cambios en el registro, lo que no permite contar con información verdadera y en tiempo real (Peña, 2004).

En general, según Donoso (2004), el caso chileno demuestra la relación directa que existe entre la escasez y el funcionamiento del mercado. Ejemplo de ello es la cuenca del río Limarí, en el sistema Pamplona, donde se presenta insuficiencia del recurso y, de igual forma, un alto valor económico, lo que genera gran competencia. En el río Maipo por otro lado, la oferta es mayor y la demanda agrícola menor, lo que ha generado algunos inconvenientes ya que los compradores y vendedores no están plenamente identificados y por lo tanto quienes participan en el mercado asumen una actitud pasiva frente a la competencia (Donoso, 2004).

Por su parte, Brown (2005) revisa la estructura institucional de la administración del agua en Chile y encuentra que hay actores públicos (Dirección Nacional de Aguas) y no públicos (organizaciones de usuarios, propietarios de embalses, generadores y trasmisores de energía eléctrica y otros usuarios como la Confederación de canalistas y organismos de cuenca). En general, el sistema de administración de los recursos hídricos en Chile es gestionado por los propios usuarios y pretende lograr una distribución del agua conforme a los derechos de aprovechamiento de cada cual. Dado que la participación de los usuarios en las elecciones de directivas y en las votaciones de la asamblea es directamente proporcional a la magnitud de los derechos de aprovechamiento de cada quien, es fácil que los mayores usuarios puedan dominar la administración y toma de decisiones en estas organizaciones (Brown, 2005).

Concluyendo con este caso, dentro de los beneficios se reconocen principalmente dos: se ha promovido la inversión en infraestructura e investigación en los diferentes usos (agrícola, industrial y generación de energía, entre otros) y a causa de la libertad para compra y venta de derechos de agua, se ha generado una reubicación del agua a los usos más productivos. En contraste, se han identificado algunas problemáticas tales como conflictos por el agua, coordinación de varios usuarios para manipular el mercado e inequidad social (Bauer, 2010).

En México, la situación es bastante particular ya que existen los mercados del agua, pero la economía de dicho recurso no se considera aún lo suficientemente madura para el desarrollo de éstos.

Esta estructura para la administración del líquido inició con la reforma legislativa que se llevó a cabo en 1992, cuando se dio vía libre a las transacciones de agua a través de concesiones de uso con una duración de treinta años. Dicha reglamentación, que se venía construyendo desde los años ochenta, dio lugar a las Asociaciones de Usuarios de Agua que pretendían organizar la administración de dichos recursos en pequeńos grupos, debido al gran número de pequeńos agricultores que había. De igual manera, se fortaleció el papel del Estado mediante la Comisión Nacional de Agua, quien hace las veces de auditor y regulador de la comercialización del recurso. Algunas personas 
manifiestan que entre mayor sea la intervención estatal, más lento será el desarrollo del mercado (R. Hearne, 1998).

González (2008) (previamente citada en el caso chileno), encontró que para México imperan los derechos adquiridos, lo que quiere decir que el sistema inicial fue tradición, es decir que los derechos se mantuvieron iguales a como estaban antes de la norma. La duración de dichos derechos es temporal de hasta treinta ańos. La transferencia ocurre por transmisiones voluntarias permanentes, el sistema de asignación en escasez es por norma proporcional, la información se suministra por agencias de transacción, el tratamiento de las externalidades por política pública reguladora y el efecto sobre las zonas de origen se realiza mediante el control público de transferencias. Los costos de transportes pueden ser tanto públicos como privados, las imperfecciones de mercado se corrigen, hay sanciones por no uso, el mecanismo para solucionar conflictos es el uso de autoridades públicas y las restricciones al mercado se realizan a través de la delimitación entre cuencas (González, 2008).

En el caso de Río San Juan, Monterrey, los derechos de agua se adjudicaron a trece Asociaciones de Usuarios de Agua y la Comisión Nacional del Agua mantiene un control constante en la adjudicación de los mismos. Dicha adjudicación se realiza teniendo en cuenta las estaciones y el clima, ya que en temporadas húmedas el agua se divide en proporción a la tierra que necesita ser regada, mientras que en épocas de sequía, se adjudica por igual entre todos los usuarios independientemente del área de riego, pero teniendo en cuenta los cultivos que son prioritarios para la economía local. En otros distritos las condiciones son parecidas; las decisiones para la adjudicación del agua están relacionadas con los factores de producción, como sucede en la Región Lagunera, en donde está prohibido el uso de aguas superficiales para el riego de alfalfa debido a que éste es un producto muy bien pago, que le permite a los agricultores responsabilizarse por el costo de la extracción de aguas subterráneas. Contrario a ello, se da prioridad al cultivo de algodón ya que hace uso intensivo de la mano de obra de la región (Hearne, 1998).

Un ejemplo en pequeña escala mencionado por Fortis \& Alhers (1999), consiste en el mercado de aguas del Distrito de Riego 017 de la Comarca Lagunera, México. Éste presentó una serie de insuficiencias relacionadas con el pequeño número de compradores que, al parecer, han creado un monopolio que influye en gran medida en los precios, los cuales no reflejan la escasez del recurso, lo que no permite contar con medios suficientes para financiar la administración e infraestructura que se requiere. Por otra parte, los derechos de agua han sido modificados constantemente a la par con un sinnúmero de eventos políticos y, además, estos se han convertido en la salida económica más fácil para aquellos pequeños comerciantes que no cuentan con recursos para abastecer un cultivo y deciden rentar sus concesiones, generando así una sobreoferta de dichos derechos (Fortis \& Alhers, 1999).

En Bolivia existen dos casos relevantes en esta materia; sin embargo empezaremos por el más significativo, debido a su trascendencia internacional y a que luego de dicha experiencia, propusieron la aplicación de una forma alternativa de administración del recurso, que no involucra entidades internacionales ni multinacionales. En Cochabamba, 
en septiembre de 1999, se dio al consorcio Aguas del Tunari, encabezado por el consorcio International Water, la concesión por cuarenta ańos del suministro de agua y saneamiento en esta ciudad. Como consecuencia, las tarifas subieron hasta un 200\% con relación a 1997. Esto representaba para los usuarios entre el $22 \%$ y el $27 \%$ de su ingreso, lo que generó enérgicas manifestaciones que dejaron como saldo la revocatoria de la concesión, un muerto, dos ciegos y varios heridos.

En segundo lugar en La Paz, capital de Bolivia, se dio la concesión a la subsidiaria Suez-Lyonnaise en 1997, quienes establecieron tarifas altas en los sectores pobres, debido a que los usuarios en dichas áreas presentaban una cultura de ahorro excesiva y no se alcanzaban las metas financieras (Barrero, 2006).

En Brasil, la privatización de la empresa Sanepar en 1998 afectó a varias regiones de Porto Alegre y Sao Paulo entre otros, cuando pasó a ser propiedad del Estado, junto con la multinacional Vivendi y el grupo brasileño Andrade Gutiérrez, apoyados, en 2001, por una fuerte inversión del Banco Mundial. En lo que está documentado se encuentra que en Itaperucu, se demostró que el agua estaba contaminada con bacterias fecales que producían enfermedades renales y diarrea; en Curitiba, la calidad del recurso era insuficiente y éste presentaba mal olor y sabor. De igual manera, en el 2001 hubo más de un evento que dejaba en duda la legalidad de la empresa, ya que se le impuso una multa de un millón de reales por explotar acuíferos subterráneos sin la licencia requerida. Concejales y autoridades ambientales le acusaron de arrojar aguas servidas impurificadas al río Vermelho y, además, existen versiones que se enmarcan en desviación de recursos, costos inflados, empleo irregular y remuneración excesiva del personal entre otros, junto con una serie de demandas por irregularidades en la facturación.

Otra experiencia tuvo lugar en Manaus, ciudad ubicada en la Amazonía brasilera, cuando se otorgó la concesión a Suez-Lyonnaise des Eaux, quienes se comprometieron a ampliar el suministro en un plazo de cinco años. El cumplimiento, sin embargo, quedó rezagado una vez se cerró la negociación debido a que la multinacional alegaba que la situación era pésima para poder hacer cambios tan pronto (Barrero, 2006).

En Cartagena, ciudad ubicada en la costa atlántica de Colombia, se llevó a cabo un proceso de privatización que no solo dejó en entre dicho las intenciones de la empresa multinacional, sino las del Gobierno, que al parecer no defendió los intereses de la comunidad, ya que se presume que la negociación se dio en un ambiente de corrupción e intereses personales. En 1995, se construyó una alianza entre el Gobierno y la empresa Aguas de Barcelona, a quienes se adjudicó la prestación del servicio de agua y saneamiento básico. Una vez iniciada su actividad, se dice que el Gobierno, a través de imposiciones militares, derrotó todo tipo de movimiento sindicalista que se desarrolló en la compañía mientras fue pública. Además, con el paso del tiempo, existieron algunas incoherencias en la información: la empresa promulgaba que la cobertura alcanzaba un $90 \%$ y en un informe del Banco Mundial, se estipulaba que casi un tercio de la población no tenía acceso a dicho recurso. Para 1999 , como una medida alternativa a la revocatoria de la concesión, que estaba ligada a la inversión del Banco Mundial, se 
subarrendó el servicio a la empresa Aquacar a cambio de un porcentaje de las ganancias (Barrero, 2006).

En el caso de Perú, en el año 2009 aprobaron la ley 29338, Ley de Recursos Hídricos, mediante la cual se establece prioridad al uso agrario del agua y a la disposición de que no existe propiedad privada sobre la misma. Dicha reforma fue resultado de varios intentos que se llevaron a cabo con antelación, pero hasta el momento no se puede hablar de los resultados (Zegarra, 2004).

España, por ser un país desarrollado, ha contado con varias iniciativas en este campo; unas de ellas de reconocido éxito y otras con grandes deficiencias. El mercado de aguas de Tenerife, aunque se destaca por haber sido un gran impulsor del crecimiento económico de la región, cuenta con una documentación que presenta algunas incoherencias según la fuente que se consulte. En un estudio realizado por Aguilera \& Sánchez (2002), se contraponen varias publicaciones: la primera corresponde al Banco Mundial a través de Simpson y Ringskog en 1997, quienes basados en las opiniones de "personas del mundo del agua", manifiestan su admiración total por el mercado del agua que allí se desarrolla; otras publicadas entre 1999 y 2001 por José Fernández Bethencourt, gerente del Consejo Insular de Aguas de Tenerife, entidad encargada del control y la auditoría del mercado del agua y, finalmente, la información recopilada por los autores a través de entrevistas y encuentros con los agricultores y habitantes de la región, quienes manifiestan opiniones completamente contrarias a lo que las autoridades mencionadas plantean en sus artículos.

Aunque son varios los puntos que resultan confusos, acá se resaltarán los que pueden resultar más polémicos. Primero, el Banco Mundial destaca la disponibilidad y amplias redes de transporte que garantizan la competitividad del mercado. Sin embargo, según Aguilera \& Sánchez, (2002) los habitantes de la región manifiestan que "los canales están controlados por los grandes vendedores de agua" y "a veces ni teniendo acciones del canal dejan pasar el agua". De igual forma, Fernández Bethencourt explica que los canales requieren modernización y reestructuración, ya que las pérdidas resultan significativas. Otra de las críticas corresponde a la afirmación de Simpson y Ringskog de que no existe necesidad de regulación debido al elevado grado de competencia que evita que se lleven a cabo abusos. Por el contrario, los pequeños accionistas manifiestan estar absolutamente indefensos y tener que vender el agua al precio que se las quieran pagar, sin contar con el sesgo en la información, que también es fruto del oligopolio que se presume se ha construido. A lo anterior, Fernández Bethencourt, quien representa a la entidad que a través del Plan Hídrico Insular está encargada de defender los derechos de los accionistas y evitar se lleven a cabo abusos, responde que la oferta es "atomizada", ya que existe un gran número de comparadores y vendedores. Esta información no se corresponde con la información suministrada por Aguilera \& Sánchez (2002), quienes demuestran que doce intermediarios poseen el 52\% del agua, de los cuales tres tienen el 26\%, lo que permite visualizar tendencias oligopólicas.

Todo lo anterior se ha visto reflejado en un aumento acelerado de accionistas que prefieren alejarse del mercado y adquirir el recurso a través de empresas de suministro, o instalando sus propias 
plantas de desalinización del mar; muestra clara de las falencias que presenta este instrumento, que en esta región parece carecer de transparencia, garantías para la competencia y confianza en el sistema.

Otro ejemplo español corresponde a Canarias, en donde se encuentran un gran número de pequeños mercados proporcionales a la cantidad de pequeños agricultores de la región. Estos funcionan mediante un sistema de arrendamiento, que se desarrolla a través de una serie de intermediarios que se convierten en el puente entre los usuarios y los accionistas, a través de un contrato que generalmente dura un año o menos, según las necesidades del cultivo, y que establece el caudal por hora que se está arrendando. En cuanto a la transacción, el demandante cancela el servicio al intermediario, quien cobra una comisión y luego realiza el pago al accionista según el precio que el agua alcance en el mercado (Ariño \& Sastre, 1997).

Aunque este tipo de mercado es un estímulo a la competencia y genera un mayor grado de conciencia frente al uso eficiente del agua, presenta los vicios más comunes como la falta de transparencia, la evasión de la legislación y la evidente prelación de algunos frente a otros.

Continuando con las experiencias europeas, Joan Pujol, Meri Raggi y Davide Viaggi realizaron un estudio juicioso de las consecuencias de la aplicación de mercados de agua en el riego de cultivos. El estudio se realizó específicamente en Foggia, Sur de Italia, país que no cuenta con una regulación clara respecto al intercambio de derechos de agua, y en Cataluña, España, donde, por el contrario, dichas transacciones están claramente reglamentadas. A través de la simulación de la asignación óptima del agua entre las diferentes granjas, asumiendo la maximización del beneficio, los autores demostraron que, gracias a la implementación de dicho instrumento, los agricultores ahorran en costos de riego, viéndose beneficiados por una mayor ganancia, la cual en Cataluña consistiría de un incremento máximo del 30\% y en Foggia máximo del 10\% (Pujol, Raggi, \& Viaggi, 2006).

De igual manera, comprobaron que esto llevaría a un mayor grado de especialización de los cultivos, ya que los derechos de agua son principalmente adquiridos por las granjas que cuentan con productos tradicionales de la región. De esta manera, en la región española se aumenta la producción de frutas y en la italiana de vegetales, generando además en los agricultores un mayor sentimiento de aprovechamiento del recurso hídrico (Zegarra, 2004).

Con base en los resultados obtenidos, no sólo se pueden ver beneficios tangibles de la aplicación de mercados del agua, sino que también para los autores queda en duda la necesidad de la implementación de subsidios a los productos agrícolas, ya que claramente se ve que el mercado los empuja al mejoramiento de sus prácticas $\mathrm{y}$, de una u otra forma, de sus ingresos (Zegarra, 2004).

\section{Conclusiones}

Autores como Lee y Juravlev (1998) respaldan la idea de que los mercados de agua funcionan bien siempre y cuando haya un conocimiento claro del marco institucional y legal y se establezcan normas y reglamentos claros con respecto a los derechos de propiedad exclusivos. Además, subrayan la necesidad de contar con mecanismos de transferencias 
sencillos y, como corolario, un mínimo de interferencia burocrática en el mercado (Lee \& Juravlev, 1998).

$\mathrm{La}$ experiencia a nivel internacional muestra que no importa el camino que se tome en cuanto al tipo de instrumento basado en el mercado, el marco institucional es determinante para su funcionamiento. En cualquier caso las externalidades siempre van a estar presentes por la naturaleza unitaria y su carácter de bien esencial. De otro lado, el repaso de la experiencia internacional también enseña que hay en general ganancias de eficiencia y mejores prácticas de asignación de recurso cuando se utilizan instrumentos de mercado (R. R. Hearne, 2007), (Garrido, 1998) y (Lee \& Juravlev, 1998). Sin embargo, este tipo de herramientas no están pensadas para servir cuando el agua se piensa desde diferentes usos o usos múltiples. Es posible que a la luz de la eficiencia no sea razonable que un agricultor use el agua del acueducto de su municipio para todas sus actividades que incluyen las productivas y las domésticas, puesto que esto impediría valorar adecuadamente el costo de oportunidad del agua. Sin embargo, una cantidad importante de campesinos integra el agua de muchas maneras a su forma de vida (Restrepo, 2010) y al separar, con fines de eficiencia, el cobro del uso para actividades productivas de las de consumo doméstico, se incrementarían significativamente sus costos de producción, de forma que se generarían conflictos sociales y distributivos que afectan la gobernabilidad del agua. Es por ello que es prudente sumarse a las palabras del profesor Griffin (2006) cuando afirma que "no single tool is a panacea" para referirse al uso generalizado de los mercados de agua como instrumento de gestión del recurso.
De otro lado, se observa a nivel internacional que los mercados de agua funcionan bien para gestionar cuando hay problemas de escasez, pues su objetivo es revelar el costo de oportunidad del uso con base en la teoría del valor. Así, aquellos que tengan exceso del recurso estarán dispuestos a ceder agua a quienes más lo valoran, de manera que ambas partes del contrato ganan. Esto ocurre en las transferencias desde el sector agrario al sector urbano. En este sentido, no se encontró ningún caso que muestre que los mercados de agua se apliquen en contextos distintos; por ejemplo para atender demandas urbanas con aguas de manantial o productos similares cuya explotación masiva puede llegar a afectar los niveles de caudales ecológicos, que son claves a la hora de mantener el equilibrio natural en el ciclo del agua.

¿Pueden ayudar los mercados de agua a resolver conflictos por el uso en nuestro país? Hay casos como los del río Guadalajara en el Valle del Cauca que serían un laboratorio. Allí, según lo analiza Moreno (2008), hay al menos dos grandes problemas: el primero es por la operación y mantenimiento del sistema de riego y la distribución del agua. El segundo, surge por la inundación y contaminación de las aguas residuales de la ciudad de Buga en predios aledaños al sitio de descarga. Para este artículo importa traer a discusión el primero, puesto que tiene que ver principalmente con agua para consumo directo y no para descarga. La situación consiste en que hay un conjunto de concesiones a productores de caña y pequeños usuarios; estas concesiones permiten al tenedor la captura de cierto caudal al ańo durante un periodo establecido. A cambio, los concesionarios pagan la llamada tasa por uso. El conflicto surge cuando llega 
la estación seca (julio-agosto) y el caudal disminuye. En ese momento, los cañeros trinchan (obstruyen) el río por las noches de tal forma que logran aumentar la cantidad tomada y regar suficientemente, pero a costa de los usuarios aguas abajo e inclusive del caudal ecológico. Adicionalmente, desde el año 1994 a la infraestructura de riego no se le hace mantenimiento puesto que la CVS (autoridad ambiental regional), por mandato de ley debía dejar de hacer dicha labor. En consecuencia, muchos tenedores de concesiones han dejado de pagar la tasa por uso porque argumentan que no reciben todo el líquido que les fue adjudicado.

Un mercado ayuda a la solución pero requiere de otros apoyos. El mercado mejoraría la eficiencia sobre todo en el tema de riego, porque se hace por gravedad y no por goteo, es decir, no hay suficientes incentivos para mejorar la tecnología. Sin embargo, se queda corto en tanto el problema radica en gran medida en que existe más que escasez física, (disponibilidad), escasez económica. Esta se manifiesta en la falta de voluntad politica, de recursos humano y económico y de coordinación entre las autoridades con competencias de manejo y protección del recurso en la cuenca, para ejercer sus funciones de manejo, protección, vigilancia y control del recurso y para invertir en la construcción y el mejoramiento de los sistemas de distribución y tratamiento del agua (Moreno, 2008, p. 77).

\section{Referencias}

I. Aguilera, F., \& Sánchez, J. (2002). Los mercados de agua en Tenerife: de la teoría a la práctica. Bakeaz: España. Ariño, G., \& Sastre, M. (1997). Los mercados del agua en Espańa: una propuesta de reforma de la Ley de aguas. Ingeniería del Agua, 4(1), 10.

2. Barrero, J. (2006). Impactos de la privatización del recurso hídrico y creación de mercados de agua: experiencias negativas en américa del sur. Unpublished Monografía, Universidad Colegio Mayor de Nuestra Señora del Rosario, Bogotá.

3. Bathla, S. (2000). Water Resource Potential in Northern India: Constraints and Analyses of Price and Non-Price Solutions. Environment, Development and Sustainability (1), 16.

4. Bauer, C. (2010). Market Approaches to Water Allocation: Lessons from Latin America. Journal. Tomado de http:// onlinelibrary.wiley.com/doi/10.1111/ j.1936-704X.2010.00073.x/abstract

5. Brennan, D. (2006). Water policy reform in Australia: lessons from the Victorian seasonal water market. Journal. Tomado de http://onlinelibrary.wiley.com/ doi/10.1111/j.1467-8489.2006.00359.x/ abstract?systemMessage $=$ There+will + be $+a+$ release+of+Wiley+Online+Library+s cheduled + for + Saturday +27 th + Novembe $\mathrm{r}+2010$ + Access+to+the+website+will+be +disrupted+as+follows\%3A+New+York+ 0630+EDT+t

6. Brewer, J., Glennon, R., Ker, A., \& Libecap, G. (2007). Presidential Address Water Markets in the West. Journal. Tomado de http://www2.bren.ucsb. edu/ -glibecap/Econ\%20Inquiry.pdf

7. Briscoe, J., \& Qamar, U. (2006). Pakistan's Water Economy Running Dry. Journal. Tomado de http://www.boellpakistan.org/downloads/Pakistans_ Water_Economy_WB.pdf

8. Brown, E. (2005). Sistema de administración del agua en Chile. En M. Ballestero, E. Brown, A. Jouravlev, U. Küffner \& E. Zegarra (Eds.), Recursos naturales e infraestructura (Vol. 90). 
Santiago de Chile: Comisión Económica Para América Latina y el Caribe CEPAL.

9. Donoso, G. (2004). Chile: estudio de caso del código de aguas. En Mercados (de derechos) de agua: experiencias y propuestas en América del Sur (pp. 25-47). Santiago de Chile: CEPAL.

ı..Easter, W., Dinar, A., \& Rosengrant, M. (1998). Water markets: Transaction Costs and Institutional options. En W. E. A. D. M. Rosengrant (Ed.), Markets for water. Potential and performance. Boston: Kluver Academic Publishers.

I I.Fortis, M., \& Alhers, R. (1999). Naturaleza y extensión del mercado del agua en el D.R. 017 de la comarca Lagunera, México. Journal, 10, 71.

I 2. Garrido, A. (1998). Economic Analysis of Water Markets in the spanish Agricultural Sector: Can they provide substantial benefits? En W. Easter, M. Rosegrant \& A. Dinar (Eds.), Markets for Water. Potential and Performance. Norwell, Massachusetts USA: Kluwer Academic Publishers.

I3.González, P. (2008). Mercados y bancos de agua: su adopción en México y restricciones insittucionales para su apliación. México: Facultad Latinoamericana de Ciencias Sociales.

I4.Grafton, R. Q., Landry, C., Libecap, G. D., McGlennon, S., \& O’Brien, R. (2010). An Integrated Assessment of Water Markets. International Center for Economic Research Disponible en: http://www.icer. it/docs/wp2010/ICERwp32-10.pdf

I 5.Australia, Chile, China, South Africa and The USA. Nber Working Paper Series (\# 13513), 56.

I6.Griffin, R. (2006). Water Resource Economics. The Analysis of Scarcity, Policies and Proyects (1 ed.). Massachusetts: MIT press.
I7. Hadjigeorgalis, E. (2009). A Place for Water Markets: Performance and Challenges. Review of Agricultural Economics, 31(1), 17.

I 8. Hearne, R. (1998). Opportunities and constrains to improved water markets in Mexico. En W. e. a. Easter (Ed.), Markets for water. Potential and performance (1 ed., pp. 13). Boston: Kluwer academic publisher.

I9. Hearne, R. R. (2007). Water markets as a mechanism for intersectoral water transfers: the Elqui Basin in Chile. Paddy Water Environment \# 5 223-227.

20. Kare Hussey, s. D. (2006). Trajectories in Australian Water Policies. Journal. Tomado de http://onlinelibrary.wiley. com/doi/10.1111/j.1936-704X.2006. mp135001005.x/abstract

2 I.Lee, T., \& Juravlev, A. (1998). Los precios, la propiedad y los mercados en la asignación del agua (Vol. 6). Santiago de Chile: Comisión económica para América Latina y el Caribe CEPAL.

22.Livingston, M. L. (1998). Institutional Requisites for Efficient Water markets. En W. Easter, M. Rosegrant \& A. Dinar (Eds.), Markets for water. Potential and performance (1 ed., pp. 15). Boston: Kluwer Academic Publishers.

23. Meinzen-Dick, R. (1998). Groundwater markets in Pakistan: Institutional development and productivity impacts. En W. e. a. Easter (Ed.), Markets for water. Potential and performance (1 ed., pp. 15). Boston: Kluwer Academic Publisher.

24. Moreno, E. (septiembre de 2008). Competencia y Conflictos de Intereses Entre Usos y Usuarios del Agua en la Cuenca Guadalajara del Valle del Cauca, Colombia. Wageningen, Holanda.

25. Peña, H. (2004). Chile 20 años del Código de Aguas. En Mercados (de derechos) de agua: experiencias y propuestas 
en América del Sur (pp. 13-24). Santiago de Chile: CEPAL.

26.Pujol, J., Raggi, M., \& Viaggi, D. (2006). The potential impact of markets for irrigation water in Italy and Spain: a comparison of two study areas. Journal. Tomado de http://onlinelibrary.wiley.com/ doi/10.1111/j.1467-8489.2006.00352.x/ abstract

27.Rawal, V. (2002). Non Market Interventions in Water Sharing Case Studies West Bengal India. Journal. Tomado de http://onlinelibrary.wiley.com/ doi/10.1111/1471-0366.00045/abstract ?systemMessage $=$ There+will+be+a+relea se+of+Wiley+Online+Library+schedule $\mathrm{d}+$ for + Saturday $+27 \mathrm{th}+$ November +2010 .+Access+to+the+website+will+be+disru pted + as+follows $\% 3 \mathrm{~A}+\mathrm{New}+$ York +0630 +EDT+to+0830+ED

28. Restrepo, I. (2010). Servicios de agua en las zonas rurales. En I,Restrepo. Usos múltiples del agua como una estrategia para la reducción de la pobreza ( $\mathrm{p}$. 232). Cali: Programa editorial Universidad del Valle.

29. Saleth, M. (1998). Water markets in India. Economic and institutional aspects.
En W. E. e. al (Ed.), Markets for water. Potential and performance (1 ed., pp. 20). Boston: Kluwer Academic publications.

30.Thobani, M. (1998). Meeting water needs in developing countries: Resolving issues in establishing tradeable water rights. En W. Easter, M. Rosegrant \& A. Dinar (Eds.), Markets for water. Potential and performance (1 ed., pp. 15). Boston: Kluwer academic Publishers.

3 I. Wachtel, H. M. (2004). Water Conflicts and International Water Markets. En Water resources in the middle east (pp. 147-154). Berlin: Springer.

32. WorldBank (2002). China, Country Water Resources Assistance Strategy. Journal. Tomado de http://siteresources.worldbank.org/INTWRD/Resources/ChinaCountryWaterResourcesAssistanceStrategy.pdf

33.Zegarra, E. (2004). Mercado de aguas: viabilidad y potencialidades de un instrumento para la reforma de la gestión hídrica en el Perú. Rega. Revista de Gestión de Agua de América Latina, 1(1), 20. 